\title{
Expansion of eigenvalues of the perturbed discrete bilaplacian
}

\author{
Shokhrukh Yu. Kholmatov ${ }^{1}$ (D) Ahmad Khalkhuzhaev² Mardon Pardabaev $^{2}$
}

Received: 17 September 2020 / Accepted: 20 January 2022 / Published online: 5 February 2022

(c) The Author(s) 2022

\section{Abstract}

We consider the family

$$
\widehat{H}_{\mu}:=\widehat{\Delta} \widehat{\Delta}-\mu \widehat{V}, \quad \mu \in \mathbb{R},
$$

of discrete Schrödinger-type operators in $d$-dimensional lattice $\mathbb{Z}^{d}$, where $\widehat{\Delta}$ is the discrete Laplacian and $\widehat{V}$ is of rank-one. We prove that there exist coupling constant thresholds $\mu_{o}, \mu^{o} \geq 0$ such that for any $\mu \in\left[-\mu^{o}, \mu_{o}\right]$ the discrete spectrum of $\widehat{H_{\mu}}$ is empty and for any $\mu \in \mathbb{R} \backslash\left[-\mu^{o}, \mu_{o}\right]$ the discrete spectrum of $\widehat{H_{\mu}}$ is a singleton $\{e(\mu)\}$, and $e(\mu)<0$ for $\mu>\mu_{o}$ and $e(\mu)>4 d^{2}$ for $\mu<-\mu^{o}$. Moreover, we study the asymptotics of $e(\mu)$ as $\mu \searrow \mu_{o}$ and $\mu \nearrow-\mu^{o}$ as well as $\mu \rightarrow \pm \infty$. The asymptotics highly depends on $d$ and $\widehat{V}$.

Keywords Discrete bilaplacian · Essential spectrum · Discrete spectrum · Eigenvalues · Asymptotics · Expansion

Mathematics Subject Classification 47A10 - 47A55 - 47A75 - 41A60

\section{Communicated by Gerald Teschl.}

Shokhrukh Yu. Kholmatov

shokhrukh.kholmatov@univie.ac.at

Ahmad Khalkhuzhaev

ahmad_x@mail.ru

Mardon Pardabaev

p_mardon75@mail.ru

1 University of Vienna, Oskar-Morgenstern Platz 1, Vienna 1090, Austria

2 Samarkand State University, University boulevard 3, Samarkand, Uzbekistan 140104 


\section{Introduction}

In this paper we investigate the spectral properties of the perturbed discrete biharmonic operator

$$
\widehat{H}_{\mu}:=\widehat{\Delta} \widehat{\Delta}-\mu \widehat{V}, \quad \mu \in \mathbb{R},
$$

in the $d$-dimensional cubical lattice $\mathbb{Z}^{d}$, where $\widehat{\Delta}$ is the discrete Laplacian and $\widehat{V}$ is a is rank-one potential with a generating potential $\widehat{v}$. This model is associated to a one-particle system in $\mathbb{Z}^{d}$ with a potential field $\widehat{v}$, in which the particle freely "jumps" from a node $X$ of the lattice not only to one of its nearest neighbors $Y$ (similar to the discrete Laplacian case), but also to the nearest neighbors of the node $Y$. From the mathematical point of view, the discrete bilaplacian represents a discrete Schrödinger operator with a degenerate bottom, i.e., $\widehat{\Delta} \widehat{\Delta}$ is unitarily equivalent to a multiplication operator by a function $\mathfrak{e}$ which behaves as $o\left(\left|p-p_{0}\right|^{2}\right)$ close to its minimum point $p_{0}$.

The spectral properties of discrete Schrödinger operators with non-degenerate bottom (i.e., e behaves as $O\left(\left|p-p_{0}\right|^{2}\right)$ close to its minimum point $\left.p_{0}\right)$, in particular with discrete Laplacian, have been extensively studied in recent years (see e.g. [1, 2, $7,8,10,11,20,21,23,26,28]$ and references therein) because of their applications in the theory of ultracold atoms in optical lattices [16, 24, 35, 36]. In particular, it is well-known that the existence of the discrete spectrum is strongly connected to the threshold phenomenon [18, 20-22], which plays an role in the existence the Efimov effect in three-body systems [31, 32, 34]: if any two-body subsystem in a three-body system has no bound state below its essential spectrum and at least two two-body subsystem has a zero-energy resonance, then the corresponding three-body system has infinitely many bound states whose energies accumulate at the lower edge of the three-body essential spectrum.

Recall that the Efimov effect may appear only for certain attractive systems of particles [29]. However, recent experimental results in the theory of ultracold atoms in an optical lattice have shown that two-particle systems can have repulsive bound states and resonances (see e.g. [36]), thus, one expects the Efimov effect to hold also for some repulsive three-particle systems in $\mathbb{Z}^{3}$.

The strict mathematical justification of the Effect effect including the asymptotics for the number of negative eigenvalues of the three-body Hamiltonian has been successfully established in 3-space dimensions (for both $\mathbb{R}^{3}$ and $\mathbb{Z}^{3}$ ) see e.g., $[1,4,13$, $19,29,31,32,34]$ and the references therein. In particular, the non-degeneracy of the bottom of the (reduced) one-particle Schrödinger operator played an important role in the study of resonance states of the associated two-body system [1, 31]. Another keypoint in the proof of the Efimov effect in $\mathbb{Z}^{3}$ was the asymptotics of the (unique) smallest eigenvalue of the (reduced) one-particle discrete Schrödinger operator which creates a singularity in the kernel of a Birman-Schwinger-type operator which used to obtain an asymptotics to the number of three-body bound states.

To the best of our knowledge, there are no published results related to the Efimov effect in lattice three-body systems in which associated (reduced) one-body Schrödinger operator has degenerate bottom. 
We also recall that fourth order elliptic operators in $\mathbb{R}^{d}$ in particular, the biharmonic operator, play also a central role in a wide class of physical models such as linear elasticity theory, rigidity problems (for instance, construction of suspension bridges) and in streamfunction formulation of Stokes flows (see e.g. [9, 25, 27] and references therein). Moreover, recent investigations have shown that the Laplace and biharmonic operators have high potential in image compression with the optimized and sufficiently sparse stored data [15]. The need for corresponding numerical simulations has led to a vast literature devoted to a variety of discrete approximations to the solutions of fourth order equations $[5,12,33]$. The question of stability of such models is basically related to their spectral properties and therefore, numerous studies have been dedicated to the numerical evaluation of the eigenvalues $[3,6,30]$.

The aim of the present paper is the study of the existence and asymptotics of eigenvalues as well as threshold resonance and bound states of $\widehat{H_{\mu}}$ defined in (1.1), which corresponds to the one-body Schrödinger operator with degenerate bottom. Namely, we study the discrete spectrum of $\widehat{H_{\mu}}$ depending on $\mu$ and on $\widehat{v}$. For simplicity we assume the generator $\widehat{v}$ of $\widehat{V}$ to decay exponentially at infinity, however, we urge that our methods can also be adjusted to less regular cases (see Remark 2.6). Since the spectrum of $\widehat{\Delta}$ consists of $[0,2 d]$ (see e.g., [1]), by the compactness of $\widehat{V}$ and Weyl's Theorem, the essential spectrum of $\widehat{H_{\mu}}$ fills the segment $\left[0,4 d^{2}\right]$ independently of $\mu$. Moreover, the essential spectrum does not give birth to a new eigenvalue while $\mu$ runs in some real interval $\left[-\mu^{o}, \mu_{o}\right]$, and it turns out as soon as $\mu$ leaves this interval through $\mu_{o}$ resp. through $-\mu^{o}$, a unique negative resp. a unique positive eigenvalue $e(\mu)$ releases from the essential spectrum (Theorem 2.2).

Now we are interested in the absorption rate of $e(\mu)$ as $\mu \rightarrow \mu_{o}$ and $\mu \rightarrow-\mu^{o}$. The associated asymptotics are highly dependent not only on the dimension $d$ of the lattice (as in the discrete Laplacian case [20,21]), but also values on the multiplicity $2 n_{o}$ and $2 n^{o}$ of $0 \in\{v=0\}$ (if $v(0)=0$ ) and $\vec{\pi} \in\{v=0\}$ (if $v(\vec{\pi})=0$ ), respectively. More precisely, depending on $d$ and $n_{o}, e(\mu)$ has a convergent expansion

- in $\left(\mu-\mu_{o}\right)^{1 / 3}$ for $2 n_{o}+d=1,7$;

- in $\mu-\mu_{o}$ for $2 n_{o}+d=3,5$;

- in $\left(\mu-\mu_{o}\right)^{1 / 4}$ for $2 n_{o}+d \geq 9$ with $d$ odd;

- in $\mu-\mu_{o}$ and $-\left(\mu-\mu_{o}\right) \ln \left(\mu-\mu_{o}\right)$ for $2 n_{o}+d=2,6$;

- in $\mu-\mu_{o}$ and $e^{-1 /\left(\mu-\mu_{o}\right)}$ for $2 n_{o}+d=4$;

- in $\left(\mu-\mu_{o}\right)^{1 / 2},-\left(\mu-\mu_{o}\right) \ln \left(\mu-\mu_{o}\right),\left(-\frac{1}{\ln \left(\mu-\mu_{o}\right)}\right)^{1 / 2}$ and $-\frac{\ln \ln \left(\mu-\mu_{o}\right)^{-1}}{\ln \left(\mu-\mu_{o}\right)}$ for $2 n_{o}+d=8$

- in $\left(\mu-\mu_{o}\right)^{1 / 2}$ and $-\left(\mu-\mu_{o}\right)^{1 / 2} \ln \left(\mu-\mu_{o}\right)$ for $2 n^{o}+d \geq 10$ with $d$ even

(see Theorem 2.4). Moreover, resonance states of 0-energy, i.e. non-zero solutions $f$ of $\widehat{H}_{\mu_{o}} f=0$ not belonging to $\ell^{2}\left(\mathbb{Z}^{d}\right)$ appear if and only if $2 n_{o}+d \in\{5,6,7,8\}$. Recall that the emergence of 0 -energy resonances in more lattice dimensions could allow the Efimov effect to be observed in other dimensions than $d=3$.

Furthermore, observing that the top $\mathfrak{e}(\vec{\pi})=4 d^{2}$ of the essential spectrum is nondegenerate, one expects the asymptotics of $e(\mu)$ as $\mu \rightarrow-\mu^{o}$ to be similar as in the discrete Laplacian case [20, 21]; more precisely, depending on $d$ and $n^{o}, e(\mu)$ has a convergent expansion

- in $\mu+\mu^{o}$ for $2 n^{o}+d=1,3$; 
- in $\left(\mu+\mu^{o}\right)^{1 / 2}$ for $2 n^{o}+d \geq 5$ with $d$ odd;

- in $\mu+\mu^{o}$ and $e^{-1 /\left(\mu+\mu^{o}\right)}$ for $2 n_{o}+d=2$;

- in $\mu+\mu^{o},-\frac{1}{\ln \left(\mu+\mu^{o}\right)}$ and $-\frac{\ln \ln \left(\mu+\mu^{o}\right)^{-1}}{\ln \left(\mu+\mu^{o}\right)}$ for $2 n_{o}+d=4$;

- in $\mu+\mu^{o}$ and $-\left(\mu+\mu^{o}\right) \ln \left(\mu+\mu^{o}\right)$ for $2 n^{o}+d \geq 6$ with $d$ even

(see Theorem 2.5). Moreover, the resonance states of energy $4 d^{2}$, i.e. non-zero solutions $f$ of $\widehat{H}_{-\mu^{o}} f=4 d^{2} f$ not belonging to $\ell^{2}\left(\mathbb{Z}^{d}\right)$ appear if and only if $2 n_{o}+d=3,4$.

The threshold analysis for more general class of nonlocal discrete Schrödinger operators with $\delta$-potential of type

$$
\widehat{H}_{\mu}=\Psi(-\widehat{\Delta})+\mu \delta_{x 0},
$$

can be found in [14], where $\Psi$ is some strictly increasing $C^{1}$-function and $\delta_{x 0}$ is the Dirac's delta-function supported at 0 . Besides the existence of eigenvalues, authors of [14] classify (embedded) threshold resonances and threshold eigenvalues depending on the behaviour of $\Psi$ at the edges of the essential spectrum of $-\widehat{\Delta}$ and on the lattice dimension $d$. The eigenvalue expansions for the discrete bilaplacian with $\delta$ perturbation have been established in [17] for $d=1$ using the complex analytic methods.

The paper is organized as follows. In Sect. 2 after introducing some preliminaries we state the main results of the paper. In Theorem 2.2 we establish necessary and sufficient conditions for non-emptiness of the discrete spectrum of $\widehat{H_{\mu}}$, and in case of existence, we study the location and the uniqueness, analiticity, monotonicity and convexity properties of eigenvalues $e(\mu)$ as a function of $\mu$. In particular, we study the asymptotics of $e(\mu)$ as $\mu \rightarrow \mu_{o}$ and $\mu \rightarrow-\mu^{o}$ as well as $\mu \rightarrow \pm \infty$. As discussed above in Theorems 2.4 and 2.5 we obtain expansions of $e(\mu)$ for small and positive $\mu-\mu_{o}$ and $\mu+\mu^{o}$. In Sect. 3 we prove the main results. The main idea of the proof is to obtain a nonlinear equation $\Delta(\mu ; z)=0$ with respect to the eigenvalue $z=e(\mu)$ of $\widehat{H_{\mu}}$ and then study properties of $\Delta(\mu ; z)$. Finally, in appendix Section A we obtain the asymptotics of certain integrals related to $\Delta(\mu ; z)$ which will be used in the proofs of main results.

\section{Data availability statement}

We confirm that the current manuscript has no associated data.

\section{Preliminary and main results}

Let $\mathbb{Z}^{d}$ be the $d$-dimensional lattice and $\ell^{2}\left(\mathbb{Z}^{d}\right)$ be the Hilbert space of squaresummable functions on $\mathbb{Z}^{d}$. Consider the family

$$
\widehat{H_{\mu}}:=\widehat{H}_{0}-\mu \widehat{V}, \quad \mu \geq 0,
$$


of self-adjoint bounded discrete Schrödinger operators in $\ell^{2}\left(\mathbb{Z}^{d}\right)$. Here $\widehat{H}_{0}:=\widehat{\Delta} \widehat{\Delta}$ is discrete bilaplacian, where

$$
\widehat{\Delta} f(x)=\frac{1}{2} \sum_{|s|=1}[f(x)-f(x+s)], \quad f \in \ell^{2}\left(\mathbb{Z}^{d}\right),
$$

is the discrete Laplacian, and $\widehat{V}$ is a rank-one operator

$$
\widehat{V} \widehat{f}(x)=\widehat{v}(x) \sum_{y \in \mathbb{Z}^{d}} \widehat{v}(y) \widehat{f}(y),
$$

where $\widehat{v} \in \ell^{2}\left(\mathbb{Z}^{d}\right) \backslash\{0\}$ is a given real-valued function.

Let $\mathbb{T}^{d}$ be the $d$-dimensional torus equipped with the Haar measure and $L^{2}\left(\mathbb{T}^{d}\right)$ be the Hilbert space of square-integrable functions on $\mathbb{T}^{d}$. By $\mathcal{F}$ we denote the the standard Fourier transform

$$
\mathcal{F}: \ell^{2}\left(\mathbb{Z}^{d}\right) \rightarrow L^{2}\left(\mathbb{T}^{d}\right), \quad \mathcal{F} \widehat{f}(p)=\frac{1}{(2 \pi)^{d / 2}} \sum_{x \in \mathbb{Z}^{d}} \widehat{f}(x) e^{i x p}
$$

Further we always assume that $\widehat{v}$ and its Fourier image

$$
v(p):=\mathcal{F} \widehat{v}(p)=\frac{1}{(2 \pi)^{d / 2}} \sum_{x \in \mathbb{Z}^{d}} \widehat{v}(x) e^{i x \cdot p}
$$

satisfy the following assumptions:

There exist reals $C, a>0$ and nonnegative integersn $n_{o}, n^{o} \geq 0$ such that

$$
\begin{aligned}
& |\widehat{v}(x)| \leq C e^{-a|x|} \quad \text { for all } x \in \mathbb{Z}^{d}, \\
& |v(0)|^{2}=D^{2}|v(0)|^{2}=\ldots=D^{2 n_{o}-2}|v(0)|^{2}=0, \quad D^{2 n_{o}}|v(0)|^{2} \neq 0, \\
& |v(\vec{\pi})|^{2}=D^{2}|v(\vec{\pi})|^{2}=\ldots=D^{2 n^{o}-2}|v(\vec{\pi})|^{2}=0, \quad D^{2 n^{o}}|v(\vec{\pi})|^{2} \neq 0,
\end{aligned}
$$

here $D^{j} f(p)$ is the $j$-th order differential of $f$ at $p$, i.e. the $j$-th order symmetric tensor

$$
\begin{gathered}
D^{j} f(p)[\underbrace{w, \ldots, w}_{j-\text { times }}]=\sum_{i_{1}+\ldots+i_{d}=j, i_{k} \geq 0} \frac{\partial^{j} f(p)}{\partial^{i_{1}} p_{1} \ldots \partial^{i_{d}} p_{d}} w_{1}^{i_{1}} \ldots w_{d}^{i_{d}}, \\
w=\left(w_{1}, \ldots, w_{d}\right) \in \mathbb{R}^{d},
\end{gathered}
$$

and $\vec{\pi}=(\pi, \ldots, \pi) \in \mathbb{T}^{d}$. Notice that under assumption $(\mathrm{H} 1), v$ is analytic on $\mathbb{T}^{d}$.

Recall that $\sigma(\widehat{\Delta})=\sigma_{\text {ess }}(\widehat{\Delta})=[0,2 d]$ (see e.g. [1]). Hence, $\sigma\left(\widehat{H}_{0}\right)=\sigma_{\text {ess }}\left(\widehat{H}_{0}\right)=$ $\left[0,4 d^{2}\right]$, and by the compactness of $\widehat{V}$ and Weyl's Theorem,

$$
\sigma_{\mathrm{ess}}\left(\widehat{H_{\mu}}\right)=\sigma_{\mathrm{ess}}\left(\widehat{H}_{0}\right)=\left[0,4 d^{2}\right]
$$


for any $\mu \in \mathbb{R}$.

Before stating the main results let us introduce the constants

$$
\begin{aligned}
& \mu_{o}:=\left(\int_{\mathbb{T}^{d}} \frac{|v(q)|^{2} d q}{\mathfrak{e}(q)}\right)^{-1}, \quad \mu^{o}:=\left(\int_{\mathbb{T}^{d}} \frac{|v(q)|^{2} d q}{4 d^{2}-\mathfrak{e}(q)}\right)^{-1}, \\
& \widehat{c}_{v}:=\int_{\mathbb{T}^{d}} \frac{|v(q)|^{2} d q}{\mathfrak{e}(q)^{2}}, \quad \widehat{C}_{v}:=\int_{\mathbb{T}^{d}} \frac{|v(q)|^{2} d q}{\left(4 d^{2}-\mathfrak{e}(q)\right)^{2}},
\end{aligned}
$$

and

$$
\begin{aligned}
c_{v} & :=\frac{2^{2 n_{o}+d}}{\left(2 n_{o}\right) !} \int_{\mathbb{S}^{d-1}} D^{2 n_{o}}|v(0)|^{2}[w, \ldots, w] d \mathcal{H}^{d-1}(w), \\
C_{v} & :=\frac{2^{2 n^{o}+d-1}}{(8 d)^{n^{o}+d / 2}\left(2 n^{o}\right) !} \int_{\mathbb{S}^{d-1}} D^{2 n^{o}}|v(\vec{\pi})|^{2}[w, \ldots, w] d \mathcal{H}^{d-1}(w),
\end{aligned}
$$

where $\mathbb{S}^{d-1}$ is the unit sphere in $\mathbb{R}^{d}$ and

$$
\mathfrak{e}(q):=\left(\sum_{i=1}^{d}\left(1-\cos q_{i}\right)\right)^{2}
$$

Remark 2.1 Under assumptions (H1)-(H3), $\mu_{o}, \mu^{o} \geq 0, c_{v}, C_{v}>0$, and $\widehat{c}_{v}, \widehat{C}_{v} \in$ $(0,+\infty]$. Moreover, by Propositions A.1 and A.2:

$-\mu_{o}=0$ (resp. $\mu^{o}=0$ ) if and only if $2 n_{o}+d \leq 4$ (resp. $2 n^{o}+d \leq 2$ );

$-\widehat{c}_{v}<\infty$ (resp. $\left.\widehat{C}_{v}<\infty\right)$ if $2 n_{o}+d \geq 9$ (resp. $2 n^{o}+d \geq 5$ ).

\subsection{Main results}

First we concern with the existence of the discrete spectrum of $\widehat{H_{\mu}}$.

Theorem 2.2 Let $\mu_{o}, \mu^{o} \geq 0$ be given by (2.2). Then $\sigma_{\text {disc }}\left(\widehat{H_{\mu}}\right)=\emptyset$ for any $\mu \in$ $\left[-\mu^{o}, \mu_{o}\right]$ and $\sigma_{\mathrm{disc}}\left(\widehat{H_{\mu}}\right)$ is a singleton $\{e(\mu)\}$ for any $\mu \in \mathbb{R} \backslash\left[-\mu^{o}, \mu_{o}\right]$. Moreover, the associated eigenfunction $\widehat{f}_{\mu}$ to $e(\mu)$ is given by $\widehat{f}_{\mu}:=\mathcal{F}^{*} f_{\mu}$, where

$$
f_{\mu}(p)=\frac{v(p)}{\mathfrak{e}(p)-e(\mu)}
$$

Furthermore, if $\mu<-\mu^{o}$ (resp. $\mu>\mu_{o}$ ), then $e(\mu)>4 d^{2}($ resp. $e(\mu)<0)$. Moreover, the function $\mu \in \mathbb{R} \backslash\left[-\mu^{o}, \mu_{o}\right] \mapsto e(\mu)$ is real-analytic strictly decreasing, convex in $\left(-\infty,-\mu^{o}\right)$ and concave in $\left(\mu_{o},+\infty\right)$, and satisfies

$$
\lim _{\mu \searrow \mu_{o}} e(\mu)=0 \quad \text { and } \quad \lim _{\mu \nearrow-\mu^{o}} e(\mu)=4 d^{2}
$$


and

$$
\lim _{\mu \rightarrow \pm \infty} \frac{e(\mu)}{\mu}=-\int_{\mathbb{T}^{d}}|v(q)|^{2} d q .
$$

Next we study the threshold resonances of $\widehat{H_{\mu}}$.

Theorem 2.3 Let $n_{o}, n^{o} \geq 0$ be given by $(H 2)-(H 3)$.

(a) Let $2 n_{o}+d \geq 5$. Then $\widehat{f}:=\mathcal{F}^{*} f \in c_{0}\left(\mathbb{Z}^{d}\right)$, i.e., $\widehat{f}(x) \rightarrow 0$ as $|x| \rightarrow+\infty$, where

$$
f(p)=\frac{v(p)}{\mathfrak{e}(p)} \in L^{1}\left(\mathbb{T}^{d}\right) .
$$

Moreover, $\widehat{f} \in c_{0}\left(\mathbb{Z}^{d}\right) \backslash \ell^{2}\left(\mathbb{Z}^{d}\right)$ for $2 n_{o}+d \in\{5,6,7,8\}, \widehat{f} \in \ell^{2}\left(\mathbb{Z}^{d}\right)$ for $2 n_{o}+d \geq 9$, and $\widehat{f}$ solves the equation $\widehat{H}_{\mu_{o}} f=0$.

(b) Let $2 n^{o}+d \geq 3$. Then $\widehat{g}:=\mathcal{F}^{*} g \in \ell^{0}\left(\mathbb{Z}^{d}\right)$, where

$$
g(p)=\frac{v(p)}{4 d^{2}-\mathfrak{e}(p)} .
$$

Moreover, $\widehat{g} \in \ell^{0}\left(\mathbb{Z}^{d}\right) \backslash \ell^{2}\left(\mathbb{Z}^{d}\right)$ for $2 n^{o}+d \in\{3,4\}, \widehat{g} \in \ell^{2}\left(\mathbb{Z}^{d}\right)$ for $2 n^{o}+d \geq 5$, and $\widehat{g}$ solves the equation $\widehat{H}_{-\mu^{o}} f=4 d^{2} f$.

We recall that in the literature the non-zero solutions of equations $\widehat{H_{\mu}} \widehat{f}=0$ and $\widehat{H_{\mu}} \widehat{g}=4 d^{2} \widehat{g}$ not belonging to $\ell^{2}\left(\mathbb{Z}^{d}\right)$ are called the resonance states $[1,2]$.

Now we study the rate of the convergences in (2.6).

Theorem 2.4 (Expansions of $e(\mu)$ at $\mu=\mu_{o}$ ) For $\mu>\mu_{o}$ let $e(\mu)<0$ be the eigenvalue of $\widehat{H_{\mu}}$.

(a) Suppose that $d$ is odd:

(al) if $2 n_{o}+d=1,3$, then $\mu_{o}=0$ and for sufficiently small and positive $\mu$,

$$
(-e(\mu))^{1 / 4}= \begin{cases}\left(\frac{\pi c_{v}}{4}\right)^{1 / 3} \mu^{1 / 3}+\sum_{n \geq 1} c_{1, n} \mu^{\frac{n+1}{3}}, & 2 n_{o}+d=1, \\ \frac{\pi c_{v}}{8} \mu+\sum_{n \geq 1} c_{3, n} \mu^{n+1}, & 2 n_{o}+d=3,\end{cases}
$$

where $\left\{c_{1, n}\right\}$ and $\left\{c_{3, n}\right\}$ are some real coefficients;

(a2) if $2 n_{o}+d=5,7$, then $\mu_{o}>0$ and for sufficiently small and positive $\mu-\mu_{o}$,

$$
\begin{aligned}
& (-e(\mu))^{1 / 4} \\
& = \begin{cases}\frac{8}{\pi c_{v} \mu_{o}^{2}}\left(\mu-\mu_{o}\right)+\sum_{n \geq 1} c_{5, n}\left(\mu-\mu_{o}\right)^{n+1}, & 2 n_{o}+d=5, \\
\left(\frac{8}{\pi c_{v} \mu_{o}^{2}}\right)^{1 / 3}\left(\mu-\mu_{o}\right)^{1 / 3}+\sum_{n \geq 1} c_{7, n}\left(\mu-\mu_{o}\right)^{\frac{n+1}{3}}, & 2 n_{o}+d=7,\end{cases}
\end{aligned}
$$

where $\left\{c_{5, n}\right\}$ and $\left\{c_{7, n}\right\}$ are some real coefficients; 
(a3) if $2 n_{o}+d \geq 9$, then $\mu_{o}>0$ and for sufficiently small and positive $\mu-\mu_{o}$,

$$
(-e(\mu))^{1 / 4}=\left(\mu_{o}^{2} \widehat{c}_{v}\right)^{-1 / 4}\left(\mu-\mu_{o}\right)^{1 / 4}+\sum_{n \geq 1} c_{9, n}\left(\mu-\mu_{o}\right)^{n / 4}
$$

where $\left\{c_{9, n}\right\}$ are some real coefficients.

(b) Suppose that $d$ is even:

(b1) if $2 n_{o}+d=2,4$, then $\mu_{o}=0$ and for sufficiently small and positive $\mu$,

$$
\begin{aligned}
& (-e(\mu))^{1 / 2} \\
& = \begin{cases}\frac{\pi c_{v}}{8} \mu+\sum_{n+m \geq 1, n, m \geq 0} c_{2, n m} \mu^{n+1}(-\mu \ln \mu)^{m}, & 2 n_{o}+d=2, \\
c e^{-\frac{8}{c_{v} \mu}}+\sum_{n+m \geq 1, n, m \geq 0} c_{4, n m} \mu^{n+1}\left(\frac{1}{\mu} e^{-\frac{8}{c_{v} \mu}}\right)^{m+1}, & 2 n_{o}+d=4,\end{cases}
\end{aligned}
$$

where $\left\{c_{2, n m}\right\}$ and $\left\{c_{4, n m}\right\}$ are some real coefficients and $c>0$;

(b2) if $2 n_{o}+d=6,8$, then $\mu_{o}>0$ and for sufficiently small and positive $\mu-\mu_{o}$,

$$
\begin{aligned}
& (-e(\mu))^{1 / 2} \\
& = \begin{cases}\frac{8}{\pi c_{v} \mu_{o}^{2}} \tau^{2}+\sum_{n+m \geq 1, n, m \geq 0} c_{6, n m} \tau^{2 n+2} \theta^{m}, & 2 n_{o}+d=6, \\
\left(\frac{8}{c_{v} \mu_{o}^{2}}\right)^{1 / 2} \tau \sigma+\sum_{n+m+k \geq 1, n, m, k \geq 0} c_{8, n m k} \tau^{n+1} \sigma^{m+1} \eta^{k}, & 2 n_{o}+d=8,\end{cases}
\end{aligned}
$$

where $\left\{c_{4, n m}\right\}$ and $\left\{c_{8, n m k}\right\}$ are some real coefficients and

$$
\tau:=\left(\mu-\mu_{o}\right)^{1 / 2}, \quad \theta:=-\tau^{2} \ln \tau, \quad \sigma:=\left(-\frac{1}{\ln \tau}\right)^{1 / 2}, \quad \eta:=-\frac{\ln \ln \tau^{-1}}{\ln \tau}
$$

(b3) if $2 n_{o}+d \geq 10$, then $\mu_{o}>0$ and for sufficiently small and positive $\mu-\mu_{o}$,

$$
(-e(\mu))^{1 / 2}=\left(\mu_{o}^{2} \widehat{c}_{v}\right)^{-1 / 2} \tau+\sum_{n+m \geq 1, n, m \geq 0} c_{10, n m} \tau^{n+1} \theta^{m},
$$

where $\left\{c_{10, n m}\right\}$ are some real coefficients.

Here $c_{v}>0$ and $\widehat{c}_{v}>0$ are given by (2.4) and (2.3), respectively.

Theorem 2.5 (Expansions of $e(\mu)$ at $\left.\mu=-\mu^{o}\right)$ For let $\mu<-\mu^{o}$ let $e(\mu)>4 d^{2}$ be the eigenvalue of $\widehat{H_{\mu}}$.

(a) Suppose that d is odd: 
(a1) if $2 n^{o}+d=1$, then $\mu^{o}=0$ and for sufficiently small and negative $\mu$,

$$
\left(e(\mu)-4 d^{2}\right)^{1 / 2}=-\pi C_{v} \mu+\sum_{n \geq 1} C_{1, n} \mu^{n+1},
$$

where $\left\{C_{1, n}\right\}$ are some real coefficients;

(a2) if $2 n^{o}+d=3$, then $\mu^{o}>0$ and for sufficiently small and positive $\mu+\mu^{o}$,

$$
\left(e(\mu)-4 d^{2}\right)^{1 / 2}=\left(\pi C_{v} \mu^{o 2}\right)^{-1}\left(\mu+\mu^{o}\right)+\sum_{n \geq 1} C_{3, n}\left(\mu+\mu^{o}\right)^{n+1},
$$

where $\left\{C_{3, n}\right\}$ and $\left\{C_{7, n}\right\}$ are some real coefficients;

(a3) if $2 n^{o}+d \geq 5$, then $\mu^{o}>0$ and for sufficiently small and positive $\mu+\mu^{o}$,

$$
\left(e(\mu)-4 d^{2}\right)^{1 / 2}=\left(\widehat{C}_{v} \mu^{o 2}\right)^{-1 / 2}\left(\mu+\mu^{o}\right)^{1 / 2}+\sum_{n \geq 1} C_{5, n}\left(\mu+\mu^{o}\right)^{(n+1) / 2},
$$

where $\left\{C_{5, n}\right\}$ are some real coefficients.

(b) Suppose that d is even:

(b1) if $2 n_{o}+d=2$, then $\mu_{o}=0$ and for sufficiently small and negative $\mu$,

$$
e(\mu)-4 d^{2}=C e^{\frac{1}{C_{v} \mu}}+\sum_{n+m \geq 1, n, m \geq 0} C_{2, n m} \mu^{n+1}\left(-\frac{1}{\mu} e^{\frac{1}{C_{v} \mu}}\right)^{m+1}
$$

where $\left\{C_{2, n m}\right\}$ are some real coefficients and $C>0$;

(b2) if $2 n_{o}+d=4$, then $\mu^{o}>0$ and for sufficiently small and positive $\mu+\mu^{o}$,

$$
e(\mu)-4 d^{2}=\left(C_{v} \mu^{o 2}\right)^{-1} \mu \sigma+\sum_{n+m+k \geq 1, n, m, k \geq 0} C_{4, n m k} \tau^{n+1} \sigma^{m+1} \eta^{k}
$$

where $\left\{C_{4, n m}\right\}$ are some real coefficients and

$$
\tau:=\mu+\mu^{o}, \quad \sigma:=-\frac{1}{\ln \tau}, \quad \eta:=-\frac{\ln \ln \tau^{-1}}{\ln \tau}
$$

(b3) if $2 n_{o}+d \geq 6$, then $\mu^{o}>0$ and for sufficiently small and positive $\mu+\mu^{o}$,

$$
\begin{aligned}
e(\mu)-4 d^{2}= & \left(\widehat{C}_{v} \mu^{o 2}\right)^{-1}\left(\mu+\mu^{o}\right) \\
& +\sum_{n+m \geq 1, n, m \geq 0} C_{6, n m}\left(\mu+\mu^{o}\right)^{n+1}\left[-\left(\mu+\mu^{o}\right) \ln \left(\mu+\mu^{o}\right)\right]^{m},
\end{aligned}
$$

where $\left\{C_{6, n m}\right\}$ are some real coefficients.

Here $C_{v}$ and $\widehat{C}_{v}$ are given by (2.5) and (2.3), respectively. 
Remark 2.6 Few comments on the main results are in order.

1. The assertions of Theorem 2.2 hold in fact for any $\widehat{v} \in \ell^{2}\left(\mathbb{Z}^{d}\right)$ (see Remark 3.2);

2. Similar expansions of $e(\mu)$ in Theorems 2.4 and 2.5 at $\mu=\mu_{o}$ and $\mu=-\mu^{o}$, respectively, still hold for any exponentially decaying $\widehat{v}: \mathbb{Z}^{d} \rightarrow \mathbb{C}$ (see Remark 3.3);

3. If $\widehat{v}$ decays at most polynomially at infinity, i.e. $\widehat{v}(x)=O\left(|x|^{-\alpha}\right)$ for some $\alpha>0$, then instead of the expansions in Theorem 2.4 and 2.5 we obtain only asymptotics of $e(\mu)$ (see Remark 3.4).

\section{Proof of main results}

In this section we prove the main results. By the Birman-Schwinger principle and the Fredholm Theorem we have

Lemma 3.1 A complex number $z \in \mathbb{C} \backslash\left[0,4 d^{2}\right]$ is an eigenvalue of $\widehat{H_{\mu}}$ if and only if

$$
\Delta(\mu ; z):=1-\mu \int_{\mathbb{T}^{d}} \frac{|v(q)|^{2} d q}{\mathfrak{e}(q)-z}=0 .
$$

Proof of Theorem 2.2 By the definition of $\mu_{o}$, for any $\mu<-\mu^{o}$ :

$$
\lim _{z \nearrow-\mu^{o}} \Delta(\mu ; z)=1+\frac{\mu}{\mu^{o}}<0, \quad \lim _{z \rightarrow+\infty} \Delta(\mu ; z)=1 .
$$

Since $\Delta(\mu ; z)>1$ for $z<0$ and $\mu>-\mu^{o}$, in view of the strict monotonicity $\Delta(\mu ; \cdot)$ in $\left(4 d^{2}, \infty\right)$, for any $\mu<-\mu^{o}$ there exists a unique $e(\mu) \in\left(4 d^{2},+\infty\right)$ such that $\Delta(\mu ; e(\mu))=0$. Analogously, for any $\mu>\mu_{o}$ there exists a unique $e(\mu) \in$ $(-\infty, 0)$ such that $\Delta(\mu ; e(\mu))=0$. By the Implicit Function Theorem the function $\mu \in \mathbb{R} \backslash\left[-\mu^{o}, \mu_{o}\right] \mapsto e(\mu)$ is real-analytic. Moreover, computing the derivatives of the implicit function $e(\mu)$ we find:

$$
e^{\prime}(\mu)=-\frac{1}{\mu} \int_{\mathbb{T}^{d}} \frac{|v(q)|^{2} d q}{\mathfrak{e}(q)-e(\mu)}\left(\int_{\mathbb{T}^{d}} \frac{|v(q)|^{2} d q}{(\mathfrak{e}(q)-e(\mu))^{2}}\right)^{-1}, \quad \mu \neq 0,
$$

thus, using $\mu(\mathfrak{e}(q)-e(\mu))>0$ we get $e^{\prime}(\mu)<0$, i.e. $e(\cdot)$ is strictly decreasing in $\mathbb{R} \backslash\{0\}$. Differentiating (3.1) one more time we get

$$
e^{\prime \prime}(\mu)=\frac{2 e^{\prime}(\mu)}{\mu}\left(1-\mu e^{\prime}(\mu) \int_{\mathbb{T}^{d}} \frac{|v(q)|^{2} d q}{(\mathfrak{e}(q)-e(\mu))^{3}}\left(\int_{\mathbb{T}^{d}} \frac{|v(q)|^{2} d q}{(\mathfrak{e}(q)-e(\mu))^{2}}\right)^{-1}\right)
$$

Therefore, $e^{\prime \prime}(\mu)>0$ (i.e. $e(\cdot)$ is strictly convex) for $\mu<0$ and $e^{\prime \prime}(\mu)<0$ (i.e. $e(\cdot)$ is strictly concave) for $\mu>0$.

To prove (2.7), first we let $\mu \rightarrow \pm \infty$ in

$$
1=\mu \int_{\mathbb{T}^{d}} \frac{|v(q)|^{2} d q}{\mathfrak{e}(q)-e(\mu)}
$$


and find $\lim _{\mu \rightarrow \pm \infty} e(\mu)=\mp \infty$. In particular, if $|\mu|$ is sufficiently large, $\left|\frac{\mathfrak{e}(q)}{e(\mu)}\right|<\frac{1}{2}$ and hence, by (3.2) and the Dominated Convergence Theorem,

$$
\lim _{\mu \rightarrow \pm \infty} \frac{e(\mu)}{\mu}=-\lim _{\mu \rightarrow \pm \infty} \int_{\mathbb{T}^{d}} \frac{|v(q)|^{2} d q}{1-\frac{\mathfrak{e}(q)}{e(\mu)}}=-\int_{\mathbb{T}^{d}}|v(q)|^{2} d q
$$

To prove that $\widehat{f_{\mu}}$ solves $\widehat{H_{\mu}} \widehat{f_{\mu}}=e(\mu) \widehat{f_{\mu}}$ we consider the equivalent equality $\mathcal{F} \widehat{H}_{\mu} \mathcal{F}^{*} f_{\mu}=e(\mu) f_{\mu}$, which is easily reduced to the equality $\Delta(\mu ; e(\mu))=0$.

Remark 3.2 In view of Lemma 3.1 and the proof of Theorem 2.2, their assertions still hold for any $v \in \ell^{2}\left(\mathbb{Z}^{d}\right)$.

Proof of Theorem 2.3 We prove only (a), the proof of (b) being similar. Repeating the proof of the continuity (resp. differentiability) of $\mathfrak{l}_{f}$ at $z=0$ in Proposition A.1 one can show that $f \in L^{1}\left(\mathbb{T}^{d}\right) \backslash L^{2}\left(\mathbb{T}^{d}\right)$ for $2 n_{o}+d \in\{5,6,7,8\}$ and $f \in L^{2}\left(\mathbb{T}^{d}\right)$ for $2 n_{o}+d \geq 9$. Thus, by the Riemann-Lebesgue Lemma, $\widehat{f} \in \ell^{0}\left(\mathbb{Z}^{d}\right)$. To show that $\widehat{H}_{\mu_{o}} \widehat{f}=0$ it suffices to observe that $\mathcal{F} \widehat{H}_{\mu_{0}} \mathcal{F}^{*} f=0$.

\section{Proof of Theorem 2.4 Since}

$$
|v(p)|^{2}=(2 \pi)^{-d}\left(\sum_{x \in \mathbb{Z}^{d}} \widehat{v}(x) \cos p \cdot x\right)^{2}+(2 \pi)^{-d}\left(\sum_{x \in \mathbb{Z}^{d}} \widehat{v}(x) \sin p \cdot x\right)^{2},
$$

the function $p \in \mathbb{T}^{d} \mapsto|v(p)|^{2}$ is nonnegative even real-analytic function. Notice also that if $n_{o} \geq 1$, then by the nonnegativity of $|v|^{2}, p=0$ is a global minimum for $|v|^{2}$. Therefore, the tensor $D^{2 n_{o}}|v(0)|^{2}$ is positively definite and

$$
c_{v}:=\frac{2^{2 n_{o}+d}}{\left(2 n_{o}\right) !} \int_{\mathbb{S}^{d-1}} D^{2 n_{o}}|v(0)|^{2}[w, \ldots, w] d \mathcal{H}^{d-1}>0 .
$$

Note that

$$
\widehat{c}_{v}=\mathfrak{l}_{|v|^{2}}^{\prime}(0)=\int_{\mathbb{T}^{d}} \frac{|v(q)|^{2} d q}{\mathfrak{e}(q)^{2}}
$$

where $\mathfrak{l}_{f}$ is defined in (A.1). By Proposition A.1, $f(p)=\frac{v(p)}{\mathfrak{e}(p)} \in L^{2}\left(\mathbb{T}^{d}\right)$ if and only if $2 n_{o}+d \geq 9$. Moreover, by definition, $\mu_{o}>0$ and $\Delta\left(\mu_{o} ; 0\right)=0$ for $2 n_{o}+d \geq 5$, and hence, as in the proof of Lemma 3.1 for such $d$ one can show that $H_{\mu_{o}} f=0$.

In view of the strict monotonicity and (2.6) there exists a unique $\mu_{1}>0$ such that $e(\mu) \in\left(-\frac{1}{128}, 0\right)$ for any $\mu \in\left(0, \mu_{1}\right)$. Since

$$
\mu=\left(\mathfrak{l}_{|v|^{2}}(e(\mu))\right)^{-1},
$$


we can use Proposition A.1 with $f=|v|^{2}$ and $e:=e(\mu)$, to find the expansions of the inverse function $\mu:=\mu(e)$. Then applying the appropriate versions of the Implicit Function Theorem in analytical case we get the expansions of $e=e(\mu)$. Notice that from (A.3) and (A.4) as well as (3.5) it follows that $\mu_{o}=0$ for $2 n_{o}+d \leq 4$ and $\mu_{o}=\left(\int_{\mathbb{T}^{d}} \frac{|v(q)|^{2} d q}{\mathfrak{e}(q)}\right)^{-1}>0$ for $2 n_{o}+d \geq 5$.

(a) Suppose that $d$ is odd. In view of the expansions (A.3) of $\mathfrak{l}_{f}$, in this case, (3.4) is reduced to the inverting the equation

$$
\mu=g(\alpha)
$$

where $\alpha:=(-e)^{1 / 4}$ and $g$ is an analytic function around $\alpha=0$.

Case $2 n_{o}+d=1$. In this case by (A.3),

$$
g(\alpha):=\frac{\alpha^{3}}{c_{1}^{3}+\sum_{n \geq 1} a_{n} \alpha^{n}},
$$

where $\left\{a_{n}\right\} \subset \mathbb{R}$ and $c_{1}:=\left(\pi c_{v} / 4\right)^{1 / 3}$ and (3.5) is equivalently represented as

$$
\alpha=\mu\left(c_{1}^{3}+\sum_{n \geq 1} a_{n} \alpha^{n}\right)^{1 / 3}
$$

where $\mu=\mu^{1 / 3}$. Now setting

$$
\alpha=\mu\left(c_{1}+u\right)
$$

and using the Taylor series of $\left(c_{1}^{3}+x\right)^{1 / 3}$, for $\mu$ and $u$ sufficiently small we rewrite (3.6) as

$$
F(u, \mu):=u-\sum_{n \geq 1} \tilde{a}_{n} \mu^{n}\left(c_{1}+u\right)^{n}=0,
$$

where $F(\cdot, \cdot)$ is analytic at $(u, \mu)=(0,0), F(0,0)=0$ and $F_{u}(0,0)=1$. Hence, by the Implicit Function Theorem, there exists $\gamma_{1}>0$ such that for $|\mu|<\gamma_{1}$, (3.8) has a unique real-analytic solution $u=u(\mu)$ which can be represented as an absolutely convergent series $u=\sum_{n \geq 1} b_{n} \mu^{n}$. Putting this in (3.7) and recalling the definitions of $\alpha$ and $\mu$ we get the expansion of $(-e(\mu))^{1 / 4}$ for $\mu>0$ small.

Case $2 n_{o}+d=3$. By (A.3),

$$
g(\alpha)=\alpha\left(c_{3}+\sum_{n \geq 1} a_{n} \alpha^{n}\right)^{-1},
$$


where $\left\{a_{n}\right\} \subset \mathbb{R}$ and $c_{3}:=\pi c_{v} / 8$, and hence, (3.5) is represented as

$$
\alpha=\mu\left(c_{3}+\sum_{n \geq 1} a_{n} \alpha^{n}\right)
$$

Then setting $\alpha=\mu\left(c_{3}+u\right)$ we rewrite (3.9) in the form (3.8), and as in the case of $2 n_{o}+d=1$, we get the expansion of $(-e(\mu))^{1 / 4}$.

Case $2 n_{o}+d=5$. In this case by (A.3)

$$
g(\alpha)=\left(\frac{1}{\mu_{o}}-\frac{\pi c_{v} \alpha}{8}\left(1+\sum_{n \geq 1} a_{n} \alpha^{n}\right)\right)^{-1}
$$

where $\left\{a_{n}\right\} \subset \mathbb{R}$, and hence, by (3.5),

$$
\frac{\mu-\mu_{o}}{\mu \mu_{o}}=\frac{\pi c_{v} \alpha}{8}\left(1+\sum_{n \geq 1} a_{n} \alpha^{n}\right)
$$

Note that if $\left|\mu-\mu_{o}\right|<\mu_{o}$, then

$$
\frac{\mu-\mu_{o}}{\mu \mu_{o}}=\frac{\mu-\mu_{o}}{\mu_{o}^{2}+\mu_{o}\left(\mu-\mu_{o}\right)}=\frac{\mu-\mu_{o}}{\mu_{o}^{2}} \sum_{n \geq 0}\left(\frac{\mu-\mu_{o}}{\mu_{o}}\right)^{n},
$$

thus from (3.10) we get

$$
\alpha=\left(\mu-\mu_{o}\right)\left(c_{5}+c_{5} \sum_{n \geq 1} \mu_{o}^{-n}\left(\mu-\mu_{o}\right)^{n}\right)\left(1+\sum_{n \geq 1} a_{n} \alpha^{n}\right)^{-1}
$$

and $c_{5}:=8 /\left(\pi c_{v} \mu_{o}^{2}\right)$. Now setting $\alpha=\left(\mu-\mu_{o}\right)\left(c_{5}+u\right)$ for sufficiently small and positive $\mu-\mu_{o}$ we get

$$
u=\sum_{n, m \geq 1} \tilde{c}_{n, m}\left(\mu-\mu_{o}\right)^{n}\left(c_{5}+u\right)^{m}
$$

where $\tilde{c}_{n, m} \subset \mathbb{R}$. By the Implicit Function Theorem, for sufficiently small $\mu-\mu_{o}$ there exists a unique real-analytic function $u=u(\mu)$ given by the absolutely convergent series $u(\mu)=\sum_{n \geq 1} b_{n}\left(\mu-\mu_{o}\right)^{n}$. By the definition of $\alpha$, this implies the expansion of $(-e(\mu))^{1 / 4}$. 
Case $2 n_{o}+d=7$. As the previous case, by (A.3) and (3.11), the equation (3.5) is represented as

$$
\left(\mu-\mu_{o}\right)\left(c_{7}^{3}+c_{7}^{3} \sum_{n \geq 1} \mu_{o}^{-n}\left(\mu-\mu_{o}\right)^{n}\right)=\alpha^{3}\left(1+\sum_{n \geq 1} a_{n} \alpha^{n}\right),
$$

where $\left\{a_{n}\right\} \subset \mathbb{R}$ and $c_{7}:=\left[8 /\left(\pi c_{v} \mu_{o}^{2}\right)\right]^{1 / 3}$. When $\mu-\mu_{o}>0$ is small enough, by the Taylor series of $(1+x)^{ \pm 1 / 3}$ at $x=0,(3.12)$ is equivalently rewritten as

$$
\alpha=\left(\mu-\mu_{o}\right)^{1 / 3}\left(c_{7}+\sum_{n \geq 1} \tilde{c}_{n}\left(\mu-\mu_{o}\right)^{n}\right)\left(1+\sum_{n \geq 1} \tilde{a}_{n} \alpha^{n}\right),
$$

Thus, for $\rho=\left(\mu-\mu_{o}\right)^{1 / 3}$, setting $\alpha=\rho\left(c_{7}+u\right)$ in (3.13), for sufficiently small and positive $\rho$ we get

$$
u=\sum_{n, m \geq 1} \tilde{c}_{n, m} \rho^{n}\left(c_{7}+u\right)^{m}
$$

By the Implicit Function Theorem, this equation has a unique real-analytic solution $u=u(\rho)$ given by the absolutely convergent series $u=\sum_{n \geq 1} b_{n} \rho^{n}$. This, definitions of $\alpha$ and $\rho$ imply the expansion of $(-e(\mu))^{1 / 4}$.

Case $2 n_{o}+d=9$. In this case by (A.3) and (3.11)

$$
\left(\mu-\mu_{o}\right)\left(c_{9}^{4}+c_{9}^{4} \sum_{n \geq 1} \mu_{o}^{-n}\left(\mu-\mu_{o}\right)^{n}\right)=\alpha^{4}\left(1+\sum_{n \geq 1} a_{n} \alpha^{n}\right),
$$

where $\left\{a_{n}\right\} \subset \mathbb{R}$ and $c_{9}:=\left(\mu_{o}^{2} \widehat{c}_{v}\right)^{-1 / 4}$. Thus, for sufficiently small and positive $\mu-\mu_{o}$ using the Taylor series of $(1+x)^{ \pm 1 / 4}$ at $x=0$, this equation can also be represented as

$$
\alpha=\rho\left(c_{9}+\sum_{n \geq 1} \tilde{b}_{n} \rho^{4 n}\right)\left(1+\sum_{n \geq 1} \tilde{a}_{n} \alpha^{n}\right)
$$

where $\rho:=\left(\mu-\mu_{o}\right)^{1 / 4}$. Now setting $\alpha=\rho\left(c_{9}+u\right)$ in (3.14) we get

$$
u=\sum_{n, m \geq 1} \tilde{c}_{n, m} \rho^{n}\left(c_{9}+u\right)^{m}
$$

and the expansion of $(-e(\mu))^{1 / 4}$ follows as in the case of $2 n_{o}+d=7$. 
(b) Suppose that $d$ is even. In view of the expansion (A.3) of $\mathfrak{l}_{f}$, in this case, (3.4) is reduced to the inverting the equation

$$
\mu=\frac{\alpha^{l}}{g(\alpha)+h(\alpha) \ln \alpha}
$$

where $\alpha:=(-e)^{1 / 2}, l \in \mathbb{N}_{0}$, and $g$ and $h$ are analytic around $\alpha=0$. Presence of $\ln \alpha$ implies that unlike the case of odd dimensions, $\alpha$ is not necessarily analytic with respect to $\mu^{s}$. Therefore, we need to introduce new variables dependent on $\ln \mu$ to reduce the problem to the Implicit Function Theorem.

Case $2 n_{o}+d=2$. By (A.4), in this case for $c_{2}:=\pi c_{v} / 8$

$$
l=1, \quad g(\alpha)=c_{2}+\sum_{n \geq 1} a_{n} \alpha^{n}, \quad h(\alpha)=\sum_{n \geq 1} b_{n} \alpha^{2 n}
$$

Hence, setting

$$
\alpha=\mu\left(c_{2}+u\right)
$$

and $\tau=-\mu \ln \mu$ we represent (3.15) as

$$
\begin{aligned}
& F(u, \mu, \tau):=u-\sum_{n \geq 1} a^{n} \mu^{n}\left(c_{2}+u\right)^{n}+\ln \left(c_{2}+u\right) \sum_{n \geq 1} b^{n} \mu^{n}\left(c_{2}+u\right)^{n} \\
& \quad-\tau \sum_{n \geq 1} b^{n} \mu^{n-1}\left(c_{2}+u\right)^{n}=0,
\end{aligned}
$$

where $F$ is analytic around $(0,0,0), F(0,0,0)=0, F_{u}(0,0,0)=1$. Hence, by the Implicit Function Theorem, there exists a unique real-analytic function $u=u(\mu, \tau)$ given by the convergent series $u(\mu, \tau)=\sum_{n+m \geq 1, n, m \geq 0} \tilde{c}_{n, m} \mu^{n} \tau^{m}$ for sufficiently small $|\mu|$ and $|\tau|$, which satisfies $F(u(\mu, \tau), \mu, \tau) \equiv 0$. Inserting $u$ in (3.16) we get the expansion of $\alpha=(-e)^{1 / 2}$.

Case $2 n_{o}+d=4$. In this case, by (A.4) for $c_{4}:=8 / c_{v}$

$$
l=0, \quad g(\alpha)=\sum_{n \geq 0} a_{n} \alpha_{n}, \quad h(\alpha)=-c_{4}+\sum_{n \geq 1} b_{n} \alpha^{2 n} .
$$

Letting $\alpha=e^{-\frac{1}{c_{4} \mu}}(c+u)$, where $c=e^{a_{0} / c_{4}}>0$, we represent (3.15) as

$$
\begin{aligned}
\ln (c+u)-b_{0} & =\frac{1}{\mu} e^{-\frac{1}{c_{4} \mu}} \sum_{n \geq 1} a^{n} e^{-\frac{n-1}{c_{4} \mu}}(c+u)^{n} \\
& +\ln (c+u) \sum_{n \geq 1} b^{n} e^{-\frac{n}{c_{4} \mu}}(c+u)^{n}-\sum_{n \geq 1} a^{n} e^{-\frac{n}{c_{4} \mu}}(c+u)^{n}=0 .
\end{aligned}
$$


Writing $\tau:=\frac{1}{\mu} e^{-\frac{1}{c_{4} \mu}}$ so that $e^{-\frac{1}{c_{4} \mu}}=\mu \tau$, (3.17) is represented as

$$
\begin{aligned}
F(u, \mu, \tau):= & \ln (c+u)-b_{0}-\mu \sum_{n \geq 1} a^{n} \mu^{n-1} \tau^{n-1}(c+u)^{n} \\
& -\ln (c+u) \sum_{n \geq 1} b^{n} \mu^{n} \tau^{n}(c+u)^{n}+\sum_{n \geq 1} a^{n} \mu^{n} \tau^{n}(c+u)^{n}=0
\end{aligned}
$$

where $F$ is analytic around $(0,0,0), F(0,0,0)=0$, and $F_{u}(0,0,0)=\frac{1}{c}>0$. Thus, by the Implicit Function Theorem, for $|\mu|,|\tau|$ and $|u|$ small there exists a unique real analytic function $u=u(\mu, \tau)$ given by the convergent series $u=$ $\sum_{n+m \geq 1, n, m \geq 0} \tilde{c}_{n, m} \mu^{n} \tau^{m}$ such that $F(u(\mu, \tau), \mu, \tau) \equiv 0$. Since $\tau=\frac{1}{\mu} e^{-\frac{1}{c_{4} \mu}}$, this implies

$$
\alpha=e^{-\frac{1}{c_{4} \mu}}(c+u)=c e^{-\frac{1}{c_{4} \mu}}+\sum_{n+m \geq 1, n, m \geq 0} \tilde{c}_{n, m} \mu^{n+1}\left(\frac{1}{\mu} e^{-\frac{1}{c_{4} \mu}}\right)^{m+1} .
$$

Case $2 n_{o}+d=6$. In this case, by (A.4), for $c_{6}:=8 /\left(\pi c_{v} \mu_{o}^{2}\right)$

$$
l=0, \quad g(\alpha)=\frac{1}{\mu_{o}}-\frac{1}{c_{6} \mu_{o}^{2}}\left(\alpha+\sum_{n \geq 2} a_{n} \alpha^{n}\right), \quad h(\alpha)=\frac{1}{c_{6} \mu_{o}^{2}} \sum_{n \geq 1} b_{n} \alpha^{2 n},
$$

and hence, (3.15) is represented as

$$
\frac{1}{\mu}-\frac{1}{\mu_{o}}=\frac{1}{c_{6} \mu_{o}^{2}}\left(\alpha+\sum_{n \geq 2} a_{n} \alpha^{n}+\ln \alpha \sum_{n \geq 1} b_{n} \alpha^{2 n}\right)
$$

or equivalently, by (3.11),

$$
\alpha=c_{6}\left(\mu-\mu_{o}\right) \sum_{n \geq 0}\left(\frac{\mu-\mu_{o}}{\mu_{o}}\right)^{n}-\sum_{n \geq 2} a_{n} \alpha^{n}-\ln \alpha \sum_{n \geq 1} b_{n} \alpha^{2 n}
$$

Recalling the definitions of $\tau$ and $\theta$ in (2.8), setting $\alpha=\tau^{2}\left(c_{6}+u\right)$, we represent (3.18) as

$$
\begin{aligned}
F(u, \tau, \theta):=u & -c_{6} \sum_{n \geq 1} \frac{\tau^{2 n}}{\mu_{o}^{n}}-\sum_{n \geq 2} a_{n} \tau^{2 n-2}\left(c_{6}+u\right)^{n} \\
& -\ln \left(c_{6}+u\right) \sum_{n \geq 1} b_{n} \tau^{4 n}\left(c_{6}+u\right)^{2 n}-\theta \sum_{n \geq 1} b_{n} \tau^{4 n-4}\left(c_{6}+u\right)^{2 n}=0,
\end{aligned}
$$


where $F$ is real-analytic around $(0,0,0), F(0,0,0)=0$ and $F_{u}(0,0,0)=1$, and $F$ is even in $\tau$. Thus, by the Implicit Function Theorem, for $|u|,|\tau|$ and $|\theta|$ small there exists a unique real analytic function $u=u(\tau, \theta)$, even in $\tau$, given by the convergent series $u=\sum_{n+m \geq 1, n, m \geq 0} \tilde{c}_{n, m} \tau^{2 n} \theta^{m}$ such that $F(u(\tau, \theta), \tau, \theta) \equiv 0$. Thus,

$$
\alpha=\tau^{2}\left(c_{6}+u\right)=c_{6} \sigma+\sum_{n+m \geq 1, n, m \geq 0} \tilde{c}_{n, m} \tau^{2 n+2} \theta^{m} .
$$

Case $2 n_{o}+d=8$. By (A.4), for $c_{8}:=\left[8 / c_{v} \mu_{o}^{2}\right]^{-1 / 2}$,

$$
l=0, \quad g(\alpha)=\frac{1}{\mu_{o}^{2} c_{8}^{2}} \sum_{n \geq 2} a_{n} \alpha^{n}, \quad h(\alpha)=\frac{1}{\mu_{o}^{2} c_{8}^{2}}\left(\alpha^{2}+\sum_{n \geq 2} b_{n} \alpha^{2 n}\right),
$$

thus, as in the case of $2 n_{o}+d=6,(3.15)$ is represented as

$$
c_{8}^{2}\left(\mu-\mu_{o}\right) \sum_{n \geq 0}\left(\frac{\mu-\mu_{o}}{\mu_{o}}\right)^{n}=\alpha^{2} \ln \alpha+\ln \alpha \sum_{n \geq 2} b_{n} \alpha^{2 n}+\sum_{n \geq 2} a_{n} \alpha^{n} .
$$

For $\tau, \sigma$ and $\eta$ given in (2.8) set $\alpha=\tau \sigma\left(c_{8}+u\right)$ and represent (3.19) as

$$
\begin{aligned}
2 c_{8} u+u^{2}= & c_{8}^{2} \sum_{n \geq 1} \frac{\tau^{2 n}}{\mu_{o}^{n}}+\sum_{n \geq 2} a_{n} \tau^{n-1} \sigma^{n+1}\left(c_{8}+u\right)^{n+2} \\
& -\sum_{n \geq 2} b_{n}(\tau \sigma)^{2 n-2}\left(c_{8}+u\right)^{2 n+2} \\
& +\left(\sigma^{2} \ln \left(c_{8}+u\right)-\frac{\eta}{2}\right)\left(\left(c_{8}+u\right)^{2}+\sum_{n \geq 2} b_{n}(\tau \sigma)^{2 n-2}\left(c_{8}+u\right)^{2 n+2}\right) .
\end{aligned}
$$

This equation is represented as $F(u, \tau, \sigma, \eta)=0$, where $F$ is real-analytic in a neighborhood of $(0,0,0,0), F(0,0,0,0)=0$ and $F_{u}(0,0,0,0)=2 c_{8}>0$. Hence, for $|u|,|\tau|,|\sigma|$ and $|\eta|$ small, by the Implicit Function Theorem, there exists a unique real-analytic function $u=u(\tau, \sigma, \eta)$ given by the convergent series $u=\sum_{n+m+k \geq 1, n, m, k \geq 0} \tilde{c}_{n, m, k} \tau^{n} \sigma^{m} \mu^{k}$ such that $F(u(\tau, \sigma, \eta), \tau, \sigma, \eta) \equiv 0$. Thus,

$$
\alpha=\tau \sigma\left(c_{8}+u\right)=c_{8} \tau \sigma+\sum_{n+m+k \geq 1, n, m, k \geq 0} \tilde{c}_{n, m, k} \tau^{n+1} \sigma^{m+1} \eta^{k}
$$

Case $2 n_{o}+d \geq 10$. By (A.4) for $c_{10}:=\left(\mu_{o}^{2} \widehat{c}_{v}\right)^{-1 / 2}$,

$$
l=0, \quad g(\alpha)=\frac{1}{\mu_{o}}+\widehat{c}_{v} \alpha^{2}+\sum_{n \geq 2} a_{n} \alpha^{n+2}, \quad h(\alpha)=\sum_{n \geq 2} b_{n} \alpha^{2 n},
$$


and as in the case of $2 n_{o}+d=6,(3.15)$ is represented as

$$
\frac{\mu-\mu_{o}}{\mu_{o}^{2}} \sum_{n \geq 0}\left(\frac{\mu-\mu_{o}}{\mu_{o}}\right)^{n}=\widehat{c}_{v} \alpha^{2}+\sum_{n \geq 2} a_{n} \alpha^{n+2}+\ln \alpha \sum_{n \geq 2} b_{n} \alpha^{2 n} .
$$

Recalling the definitions of $\tau$ and $\theta$ in (2.8), we set $\alpha=\tau\left(c_{10}+u\right)$. Then (3.20) is represented as

$$
\begin{aligned}
F(u, \tau, \theta):= & 2 c_{10} u+u^{2}-c_{10}^{2} \sum_{n \geq 1} \frac{\tau^{2 n}}{\mu_{o}^{n}}+\sum_{n \geq 2} a_{n} \tau^{n}\left(c_{10}+u\right)^{n+2} \\
& -\theta \sum_{n \geq 2} b_{n} \tau^{2 n-4}\left(c_{8}+u\right)^{2 n}+\ln \left(c_{10}+u\right) \sum_{n \geq 2} b_{n} \tau^{2 n-2}\left(c_{8}+u\right)^{2 n}=0,
\end{aligned}
$$

where $F$ is analytic at $(0,0,0), F(0,0,0)=0$ and $F_{u}(0,0,0)=2 c_{10}>0$. Thus, by the Implicit Function Theorem, for $|u|,|\tau|$ and $|\theta|$ small there exists a unique real-analytic function $u=u(\tau, \theta)$ given by the convergent series $u=$ $\sum_{n+m \geq 1, n, m \geq 0} \tilde{c}_{n, m} \tau^{n} \theta^{n}$ such that $F(u(\tau, \theta), \tau, \theta) \equiv 0$. Then

$$
\alpha=\mu\left(c_{10}+u\right)=c_{10} \mu+\sum_{n+m \geq 1, n, m \geq 0} \tilde{c}_{n, m} \mu^{n+1} \theta^{n} .
$$

Theorem is proved.

Proof of Theorem 2.5 From (3.3) it follows that the map $p \in \mathbb{T}^{d} \mapsto|v|^{2}(\vec{\pi}+p)$ is even. Now the expansions of $e(\mu)$ at $\mu=-\mu^{o}$ can be proven along the same lines of Theorem 2.4 using Proposition A.2 with $f=|v|^{2}$.

Remark 3.3 Let $\widehat{v}: \mathbb{Z}^{d} \rightarrow \mathbb{C}$ satisfy $(\mathrm{H} 1)$. Since $\mathfrak{e}(\cdot)$ is even,

$$
\int_{\mathbb{T}^{d}} \frac{|v(p)|^{2} d p}{\mathfrak{e}(p)-z}=\frac{1}{(2 \pi)^{d}} \int_{\mathbb{T}^{d}} \frac{f(p) d p}{\mathfrak{e}(p)-z}
$$

where

$$
\begin{aligned}
f(p):= & \left(\sum_{x \in \mathbb{Z}^{d}} \widehat{v}_{1}(x) \cos p \cdot x\right)^{2}+\left(\sum_{x \in \mathbb{Z}^{d}} \widehat{v}_{2}(x) \cos p \cdot x\right)^{2} \\
& +\left(\sum_{x \in \mathbb{Z}^{d}} \widehat{v}_{1}(x) \sin p \cdot x\right)^{2}+\left(\sum_{x \in \mathbb{Z}^{d}} \widehat{v}_{2}(x) \sin p \cdot x\right)^{2}
\end{aligned}
$$


and $\widehat{v}=\widehat{v}_{1}+i \widehat{v}_{2}$ for some $\widehat{v}_{1}, \widehat{v}_{2}: \mathbb{Z}^{d} \rightarrow \mathbb{R}$. By Lemma 3.1, the unique eigenvalue $e(\mu)$ of $H_{\mu}$ solves

$$
1-\mu \int_{\mathbb{T}^{d}} \frac{f(p) d p}{\mathfrak{e}(p)-e(\mu)}=0
$$

Since both $p \in \mathbb{T}^{d} \mapsto f(p)$ and $p \in \mathbb{T}^{d} \mapsto f(\vec{\pi}+p)$ are even analytic functions, we can still apply Propositions A.1 and A.2 to find the expansions of $z \mapsto \int_{\mathbb{T}^{d}} \frac{f(p) d p}{\mathfrak{e}(p)-z}$ and thus, repeating the same arguments of the proofs of Theorems 2.4 and 2.5 one can obtain the corresponding expansions of $e(\mu)$.

Remark 3.4 When

$$
|\widehat{v}(x)|=O\left(|x|^{2 n_{0}+d+1}\right) \quad \text { as }|x| \rightarrow \infty
$$

for some $n_{0} \geq 1$, in view of Remark A.3, we need to solve equation (3.4) with respect to $\mu$ using only that left-hand side is an asymptotic sum (not a convergent series). This still can be done using appropriate modification of the Implicit Function Theorem for differentiable functions. As a result, we obtain only (Taylor-type) asymptotics of $e(\mu)$.

Acknowledgements Sh. Kholmatov acknowledges support from the Austrian Science Fund (FWF) project M 2571-N32.

Funding Open access funding provided by University of Vienna.

Open Access This article is licensed under a Creative Commons Attribution 4.0 International License, which permits use, sharing, adaptation, distribution and reproduction in any medium or format, as long as you give appropriate credit to the original author(s) and the source, provide a link to the Creative Commons licence, and indicate if changes were made. The images or other third party material in this article are included in the article's Creative Commons licence, unless indicated otherwise in a credit line to the material. If material is not included in the article's Creative Commons licence and your intended use is not permitted by statutory regulation or exceeds the permitted use, you will need to obtain permission directly from the copyright holder. To view a copy of this licence, visit http://creativecommons.org/licenses/by/4.0/.

\section{Appendix A. Asymptotics of some integrals}

In this section we study the behaviour of the integral

$$
\mathfrak{l}_{f}(z):=\int_{\mathbb{T}^{d}} \frac{f(q) d q}{\mathfrak{e}(q)-z}, \quad z \in \mathbb{C} \backslash\left[0,4 d^{2}\right],
$$

as $z \rightarrow 0$ and $z \rightarrow 4 d^{2}$, where $f: \mathbb{T}^{d} \rightarrow \mathbb{R}$ is a real-analytic even function on $\mathbb{T}^{d}$. Further we denote by $W_{r}(\xi) \subset \mathbb{C}$ the complex disc of radius $r>0$ centered at $\xi \in \mathbb{C}$.

Proposition A.1 Let $f: \mathbb{T}^{d} \rightarrow \mathbb{R}$ be a real-analytic even function such that

$$
f(0)=D^{2} f(0)=\ldots=D^{2 n_{o}-2} f(0)=0, \quad D^{2 n_{o}}(0) \neq 0
$$


for some $n_{o} \geq 0$. Then:

$-\mathfrak{l}_{f}$ is continuous at 0 if and only if $2 n_{o}+d \geq 5$;

$-\mathfrak{l}_{f}$ is continuously differentiable at 0 if and only if $2 n+d \geq 9$, in this case,

$$
\mathfrak{l}_{f}^{\prime}(0):=\int_{\mathbb{T}^{d}} \frac{f(q) d q}{(\mathfrak{e}(q))^{2}}=\lim _{z \searrow 0} \int_{\mathbb{T}^{d}} \frac{f(q) d q}{(\mathfrak{e}(q)-z)^{2}}
$$

Moreover, for any $z \in\left(-\frac{1}{64}, 0\right)$ :

(a) if $d$ is odd, then

$$
\mathfrak{l}_{f}(z)= \begin{cases}\frac{\pi}{4(-z)^{3 / 4}}\left(c_{f}+\sum_{n \geq 1} a_{n}^{d}(-z)^{n / 4}\right), & 2 n_{o}+d=1, \\ \frac{\pi}{8(-z)^{1 / 4}}\left(c_{f}+\sum_{n \geq 1} a_{n}^{d}(-z)^{n / 4}\right), & 2 n_{o}+d=3, \\ \mathfrak{l}_{f}(0)-\frac{\pi(-z)^{1 / 4}}{8}\left(c_{f}+\sum_{n \geq 1} a_{n}^{d}(-z)^{n / 4}\right), & 2 n_{o}+d=5, \\ \mathfrak{l}_{f}(0)-\frac{\pi(-z)^{3 / 4}}{8}\left(c_{f}+\sum_{n \geq 1} a_{n}^{d}(-z)^{n / 4}\right), & 2 n_{o}+d=7, \\ \mathfrak{l}_{f}(0)+z\left(\mathfrak{l}_{f}^{\prime}(0)+\sum_{n \geq 1} a_{n}^{d}(-z)^{n / 4}\right), & 2 n_{o}+d \geq 9,\end{cases}
$$

(b) if $d$ is even, then

$$
\mathfrak{l}_{f}(z)= \begin{cases}\frac{\pi}{8(-z)^{1 / 2}}\left(c_{f}+\sum_{n \geq 1} b_{n}^{d}(-z)^{n / 2}\right)-\frac{1}{16} \ln (-z) \sum_{n \geq 0} c_{n}^{d} z^{n}, & 2 n_{o}+d=2, \\ -\frac{1}{16} \ln (-z)\left(c_{f}+\sum_{n \geq 1} c_{n}^{d} z^{n}\right)+\sum_{n \geq 0} b_{n}^{d}(-z)^{n / 2}, & 2 n_{o}+d=4, \\ \mathfrak{l}_{f}(0)-\frac{\pi(-z)^{1 / 2}}{8}\left(c_{f}+\sum_{n \geq 1} b_{n}^{d}(-z)^{n / 2}\right)+z \ln (-z) \sum_{n \geq 0} c_{n}^{d} z^{n}, & 2 n_{o}+d=6, \\ \mathfrak{l}_{f}(0)-\frac{z}{16} \ln (-z)\left(c_{f}+\sum_{n \geq 1} c_{n}^{d} z^{n}\right)+\sum_{n \geq 2} b_{n}^{d}(-z)^{n / 2}, & 2 n_{o}+d=8 \\ \mathfrak{l}_{f}(0)+z\left(\mathfrak{l}_{f}^{\prime}(0)+\sum_{n \geq 1} b_{n}^{d}(-z)^{n / 2}\right)+z^{2} \ln (-z) \sum_{n \geq 0} c_{n}^{d} z^{n}, & 2 n_{o}+d \geq 10\end{cases}
$$

where $\left\{a_{n}^{d}\right\},\left\{b_{n}^{d}\right\}$ and $\left\{c_{n}^{d}\right\}$ are some real coefficients,

$$
c_{f}:=\frac{2^{2 n_{o}+d}}{\left(2 n_{o}\right) !} \int_{\mathbb{S}^{d-1}} D^{2 n_{o}} f(0)[w, \ldots, w] d \mathcal{H}^{d-1}
$$

and all series in (A.3) and (A.4) converge absolutely for $z \in W_{1 / 64}(0) \subset \mathbb{C}$. 
Proof Given $\gamma \in\left(0, \frac{1}{\sqrt{2}}\right]$, let $\varphi: B_{\gamma}(0) \subset \mathbb{R}^{d} \rightarrow \varphi\left(B_{\gamma}(0)\right) \subset \mathbb{R}^{d}$ be the smooth diffeomorphism

$$
\varphi_{i}(y)=2 \arcsin y_{i}, \quad i=1, \ldots, d .
$$

Note that

$$
\mathfrak{e}(\varphi(y))=\left(\sum_{i=1}^{d}\left(1-\cos \left(2 \arcsin \left(y_{i}\right)\right)\right)\right)^{2}=4\left(\sum_{i=1}^{d} y_{i}^{2}\right)^{2}=4 y^{4},
$$

therefore,

$$
\mathfrak{e}(q) \geq 4 \gamma^{4} \quad \text { for any } q \in \mathbb{T}^{d} \backslash \varphi\left(B_{\gamma}\right) .
$$

We rewrite $\mathfrak{l}_{f}(z)$ as

$$
\mathfrak{l}_{f}(z):=\int_{\varphi\left(B_{\gamma}(0)\right)} \frac{f(q) d q}{\mathfrak{e}(q)-z}+\int_{\mathbb{T}^{d} \backslash \varphi\left(B_{\gamma}(0)\right)} \frac{f(q) d q}{\mathfrak{e}(q)-z}:=\mathfrak{l}^{*}(z)+\mathfrak{l}^{* *}(z)
$$

By virtue of (A.7),

$$
\mathfrak{l}^{* *}(z)=\int_{\mathbb{T}^{d} \backslash \varphi\left(B_{\gamma}(0)\right)} \frac{f(q)}{\mathfrak{e}(q)}\left(1-\frac{z}{\mathfrak{e}(q)}\right)^{-1} d q=\sum_{n \geq 0} z^{n} \int_{\mathbb{T}^{d} \backslash \varphi\left(B_{\gamma}(0)\right)} \frac{f(q) d q}{(\mathfrak{e}(q))^{n+1}}
$$

i.e. $\mathfrak{l}^{* *}(\cdot)$ is analytic in $W_{2 \gamma^{4}}(0)$. In $\mathfrak{r}^{*}$ making the change of variables $q=\varphi(y)$ and using (A.6) we get

$$
\mathfrak{l}^{*}(z)=\int_{B_{\gamma}(0)} \frac{f(\varphi(y)) J(\varphi(y)) d y}{4 y^{4}-z}
$$

where $y^{4}:=\left(y^{2}\right)^{2}$ with $y^{2}:=\sum_{i=1}^{d} y_{i}^{2}$, and

$$
J(\varphi(y))=\prod_{i=1}^{d} \frac{2}{\sqrt{1-y_{i}^{2}}}
$$

is the Jacobian of $\varphi$. Since $f$ is an even analytic function satisfying (A.2), even each coordinate, from the Taylor series for $f$ it follows that

$$
f(p)=\sum_{n \geq n_{o}} \frac{1}{(2 n) !} D^{2 n} f(0)[\underbrace{p, \ldots, p}_{2 n \text {-times }}],
$$


and by the analyticity of $f$ in $B_{\pi}(0) \subset \mathbb{R}^{d}$, the series converges absolutely in $p \in B_{\pi}(0)$. By the definition of $\varphi, \varphi(r w) \subset B_{\pi}(0)$ for any $r \in(0, \gamma)$ and $w=\left(w_{1}, \ldots, w_{d}\right) \in \mathbb{S}^{d-1}$, where $\mathbb{S}^{d-1}$ is the unit sphere in $\mathbb{R}^{d}$. Then letting $p=\varphi(r w)$ and using the Taylor series

$$
\varphi_{i}(r w)=2 r w_{i}+\frac{r^{3} w_{i}^{3}}{3}+\sum_{n \geq 3} \tilde{c}_{n} r^{2 n-1} w_{i}^{2 n-1}
$$

of $2 \arcsin (\cdot)$, which is absolutely convergent for $\left|r w_{i}\right|<1$, from (A.11) we obtain

$$
f(\varphi(r w))=\sum_{n \geq n_{o}} \tilde{C}_{n}(w) r^{2 n}
$$

where $\tilde{C}_{n}: \mathbb{S}^{d-1} \rightarrow \mathbb{R}$ is a homogeneous polynomial of $w \in \mathbb{S}^{d-1}$ of degree $2 n$, and

$$
\tilde{C}_{n_{o}}(w)=\frac{2^{2 n_{o}}}{\left(2 n_{o}\right) !} D^{2 n_{o}} f(0)[\underbrace{w, \ldots, w}_{2 \mathrm{n}_{o}-\text { times }}]
$$

Next consider $J(\varphi(y))$. Inserting the Taylor series of $(1-t)^{-1 / 2}$ into (A.10) we obtain

$$
J(\varphi(r w))=2^{d}\left(1+\sum_{n \geq 1} \widehat{C}_{n}(w) r^{2 n}\right)
$$

where $\widehat{C}_{n}: \mathbb{S}^{d-1} \rightarrow \mathbb{R}$ is a homogeneous symmetric polynomial of $w \in \mathbb{S}^{d-1}$ of degree $2 n$, and the series converges absolutely.

Now passing to polar coordinates by $y=r w$ in (A.9) and using (A.12) and (A.13) as well as the absolute convergence of the series we get

$$
r^{*}(z)=2^{d} \int_{0}^{\gamma} \frac{r^{d-1}}{4 r^{4}-z}\left(\sum_{n \geq n_{o}} \int_{\mathbb{S}^{d-1}} C_{n}(w) r^{2 n}\right) d \mathcal{H}^{d-1} d r=\sum_{n \geq n_{o}} \widehat{c}_{n} \int_{0}^{\gamma} \frac{r^{2 n+d-1} d r}{4 r^{4}-z}
$$

where $C_{n}: \mathbb{S}^{d-1} \rightarrow \mathbb{R}$ is a homogeneous polynomial of $w \in \mathbb{S}^{d-1}$ of degree $2 n$ and

$$
\widehat{c}_{n}:=2^{d} \int_{\mathbb{S}^{d-1}} C_{n}(w) d \mathcal{H}^{d-1} .
$$

Note that $\widehat{c}_{n_{o}}=c_{f}$, where $c_{f}$ is given by (A.5) and the last series in (A.14) uniformly converges in any compact subset of $\mathbb{C} \backslash[0,4]$ since $\mathfrak{I}^{*}$ and

$$
z \in \mathbb{C} \backslash[0,4] \mapsto \mathfrak{j}_{2 n+d-1}(z):=\int_{0}^{\gamma} \frac{r^{2 n+d-1} d r}{4 r^{4}-z}
$$


are analytic functions in $\mathbb{C} \backslash[0,4]$ and all series in (A.14) converge pointwise ${ }^{1}$. Note that for any $m \geq 0$, there exist $c_{m} \in \mathbb{R}$ and an analytic function $f_{m}$ in the ball $W_{\gamma^{4}}(0) \subset \mathbb{C}$ such that for any $z \in\left(-\gamma^{4}, 0\right)$,

$$
\mathfrak{j}_{m}(z)=z^{n} \mathfrak{j}_{l}^{o}(z)+c_{m}+z^{v} f_{m}\left((-z)^{1 / 2}\right),
$$

where $n:=\left[\frac{m}{4}\right], l:=m-4 n \in\{0,1,2,3\}, v=\frac{1}{2}$ for $m=0,2$ and $v=1$ for $m=1,3$ or $m \geq 4$, and

$$
\mathfrak{j}_{l}^{o}(z):= \begin{cases}\frac{\pi}{4}(-z)^{-3 / 4} & \text { if } l=0, \\ \frac{\pi}{8}(-z)^{-1 / 2} & \text { if } l=1, \\ \frac{\pi}{8}(-z)^{-1 / 4} & \text { if } l=2, \\ -\frac{1}{16} \ln (-z) & \text { if } l=3 .\end{cases}
$$

Inserting (A.15) into (A.14) we obtain

$$
\begin{aligned}
\mathfrak{l}^{*}(z)=\sum_{n \geq n_{o}} & \widehat{c}_{n}\left(z^{\left[\frac{2 n+d-1}{4}\right]} \mathfrak{j}_{2 n+d-1-4\left[\frac{2 n+d-1}{4}\right]}^{o}(z)\right. \\
& \left.+c_{2 n+d-1}+\widehat{c}_{n}(-z)^{v_{n}} f_{2 n+d-1}\left((-z)^{1 / 2}\right)\right),
\end{aligned}
$$

where $\left\{c_{2 n+d-1}\right\} \subset \mathbb{R}$ and $\left\{f_{2 n+d-1}\right\}$ is a sequence of analytic functions in $W_{\gamma^{4}}(0)$ and

$$
v_{n}:= \begin{cases}\frac{1}{2}, & 2 n+d=1,3 \\ 1, & \text { otherwise. }\end{cases}
$$

Since (A.14) converges locally uniformly in $\mathbb{C} \backslash[0,4], C:=\sum_{n \geq n_{o}} \widehat{c}_{n} c_{2 n+d-1}$ is finite and

$$
\sum_{n \geq n_{o}} \widehat{c}_{n}(-z)^{v_{n}} f_{2 n+d-1}\left((-z)^{1 / 2}\right)=(-z)^{v} g\left((-z)^{1 / 2}\right),
$$

where $g$ is analytic in $W_{\gamma^{2}}(0)$ and $v=\frac{1}{2}$ for $2 n_{o}+d=1,3$ and $v=1$ otherwise. Hence,

$$
\mathfrak{l}^{*}(z)=C+(-z)^{v} g\left((-z)^{1 / 2}\right)+\sum_{n \geq n_{o}} \widehat{c}_{n} z^{\left[\frac{2 n+d-1}{4}\right]} \mathfrak{j}_{2 n+d-1-4\left[\frac{2 n+d-1}{4}\right]}^{o}(z),
$$

\footnotetext{
${ }^{1}$ If $\left\{h_{n}\right\}$ is an equi-bounded sequence of analytic functions in a connected open set $\Omega \subset \mathbb{C}$ converging pointwise to a function $h: \Omega \rightarrow \mathbb{C}$, then $h$ is analytic and $h_{n}$ converges uniformly to $h$ in compact subsets of $\Omega$.
} 
If $0 \leq 2 n_{o}+d-1 \leq 3$, then by (A.16),

$$
\begin{aligned}
\mathfrak{l}^{*}(z) & =C+(-z)^{v} g\left((-z)^{1 / 2}\right)+\widehat{c}_{n_{o}} \mathfrak{j}_{2 n_{o}+d-1}^{o}(z) \\
& +\sum_{n \geq n_{o}+1} \widehat{c}_{n} z^{\left[\frac{2 n+d-1}{4}\right]} \mathfrak{j}_{2 n+d-1-4\left[\frac{2 n+d-1}{4}\right]}^{o}(z)
\end{aligned}
$$

In view of (A.8) and the definition of $\mathfrak{j}_{l}^{o}$, from (A.17) we obtain the expansions (A.3) and (A.4) of $\mathfrak{l}_{f}$ for $2 n_{o}+d \leq 4$. In particular, since $\left[\frac{2 n+d-1}{4}\right] \geq 1$ for $n \geq n_{o}+1$, letting $z \rightarrow 0$ in (A.17) we get

$$
\lim _{z \rightarrow 0} \mathfrak{l}^{*}(z)=+\infty
$$

If $2 n_{o}+d-1 \geq 4$, then $\left[\frac{2 n+d-1}{4}\right] \geq 1$ for any $n \geq n_{o}$. Therefore, by (A.16), $\mathfrak{l}^{*}(0):=\lim _{z \rightarrow 0} \mathfrak{l}^{*}(z)$ exists and equals to $C$. In particular, for $2 n_{o}+d-1 \leq 7$, one has

$$
\begin{aligned}
\mathfrak{l}^{*}(z) & =\mathfrak{l}^{*}(0)-z g\left((-z)^{1 / 2}\right)+\widehat{c}_{n_{o}} z \mathfrak{j}_{2 n_{o}+d-1}^{o}(z) \\
& +\sum_{n \geq n_{o}+1} \widehat{c}_{n} z^{\left[\frac{2 n+d-1}{4}\right]} \mathfrak{j}_{2 n+d-1-4\left[\frac{2 n+d-1}{4}\right]}^{o}(z)
\end{aligned}
$$

from which and (A.8) we deduce the expansions (A.3) and (A.4) of $\mathfrak{l}_{f}$ for $5 \leq 2 n_{o}+d \leq$ 8. In particular, by virtue of (A.18) and analyticity of $\mathfrak{l}^{* *}$ at $z=0, \mathfrak{l}_{f}$ is continous at 0 if and only if $2 n_{o}+d \geq 5$. Notice also by (A.19)

$$
\lim _{z \rightarrow 0} \frac{\mathfrak{l}^{*}(z)-\mathfrak{l}^{*}(0)}{z}=+\infty
$$

i.e. $\mathfrak{l}^{*}$ (and hence $\mathfrak{l}_{f}$ ) is not differentiable at $z=0$.

Finally, if $2 n_{o}+d-1 \geq 8$, then $\left[\frac{2 n+d-1}{4}\right] \geq 2$ for any $n \geq n_{o}$. Therefore, by (A.16) there exists

$$
\mathfrak{l}^{* \prime}(0):=\lim _{z \rightarrow 0} \frac{\mathfrak{l}^{*}(z)-\mathfrak{l}^{*}(0)}{z}=-g(0) .
$$

Now using the Taylor series of $g$ at 0 we get

$$
z g\left((-z)^{1 / 2}\right)=\mathfrak{l}^{* \prime}(0) z+z \sum_{n \geq 1} \frac{g^{(n)}(0)}{n !}(-z)^{n / 2} .
$$

Inserting this in (A.16), using the definition of $\mathfrak{j}_{l}^{o}$ and the analyticity of $\mathfrak{l}^{* *}$ we get the expansions (A.3) and (A.4) of $\mathfrak{l}_{f}$ for $2 n_{o}+d \geq 9$.

By (A.18) and (A.20), $\mathfrak{l}_{f}$ is continously differentiable at 0 if and only if $2 n_{o}+d \geq 9$.

Now the choice $\gamma=\frac{1}{\sqrt{2}}$ completes the proof. 
Proposition A.2 Let $f: \mathbb{T}^{d} \rightarrow \mathbb{R}$ be a real-analytic function such that $q \in \mathbb{T}^{d} \mapsto$ $f(\vec{\pi}+q)$ is even and

$$
f(\vec{\pi})=D^{2} f(\vec{\pi})=\ldots=D^{2 n_{o}-2} f(\vec{\pi})=0, \quad D^{2 n_{o}}(\vec{\pi}) \neq 0
$$

for some $n_{o} \in \mathbb{N}_{0}$. Then:

$-\mathfrak{l}_{f}$ is continuous at $z=4 d^{2}$ if and only if for $2 n_{o}+d \geq 3$,

$-\mathfrak{l}_{f}$ is continuously differentiable at $z=4 d^{2}$ if and only if for $2 n_{o}+d \geq 5$, in this case

$$
\mathfrak{l}_{f}^{\prime}\left(4 d^{2}\right):=\int_{\mathbb{T}^{d}} \frac{f(q) d q}{\left(\mathfrak{e}(q)-4 d^{2}\right)^{2}}=\lim _{z \searrow 4 d^{2}} \int_{\mathbb{T}^{d}} \frac{f(q) d q}{(\mathfrak{e}(q)-z)^{2}}
$$

exists.

Moreover, if $z-4 d^{2} \in\left(0, \frac{1}{16}\right), \mathfrak{l}_{f}(z)$ is represented as:

(a) if $d$ is odd, then

$$
\mathfrak{l}_{f}(z)= \begin{cases}-\frac{\pi C_{f}}{\sqrt{z-4 d^{2}}}+\sum_{k \geq 0} a_{k}^{d}\left(z-4 d^{2}\right)^{k / 2}, & 2 n_{o}+d=1 \\ \mathfrak{l}_{f}\left(4 d^{2}\right)+\pi C_{f} \sqrt{z-4 d^{2}}+\sum_{k \geq 2} a_{k}^{d}\left(z-4 d^{2}\right)^{k / 2}, & 2 n_{o}+d=3 \\ \mathfrak{l}_{f}\left(4 d^{2}\right)+\mathfrak{l}_{f}^{\prime}\left(4 d^{2}\right)\left(z-4 d^{2}\right)+\sum_{k \geq 3} a_{k}^{d}\left(z-4 d^{2}\right)^{k / 2}, & 2 n_{o}+d \geq 5\end{cases}
$$

(b) if $d$ is even, then

$$
\mathfrak{l}_{f}(z)= \begin{cases}C_{f} \ln \alpha+\ln \alpha \sum_{k \geq 1} b_{k}^{d} \alpha^{k}+\sum_{k \geq 0} c_{k}^{d} \alpha^{k}, & 2 n_{o}+d=2, \\ \mathfrak{l}_{f}\left(4 d^{2}\right)-C_{f} \alpha \ln \alpha+\ln \alpha \sum_{k \geq 2} b_{k}^{d} \alpha^{k}+\sum_{k \geq 1} c_{k}^{d} \alpha^{k}, & 2 n_{o}+d=4, \\ \mathfrak{l}_{f}\left(4 d^{2}\right)+\mathfrak{l}_{f}^{\prime}\left(4 d^{2}\right) \alpha+\ln \alpha \sum_{k \geq 2} b_{k}^{d} \alpha^{k}+\sum_{k \geq 2} c_{k}^{d} \alpha^{k}, & 2 n_{o}+d \geq 6,\end{cases}
$$

where $\alpha:=z-4 d^{2},\left\{a_{k}^{d}\right\},\left\{b_{k}^{d}\right\},\left\{c_{k}^{d}\right\} \subset \mathbb{R}$ and

$$
C_{f}:=\frac{2^{2 n_{o}+d-1}}{(8 d)^{n_{o}+d / 2}\left(2 n_{o}\right) !} \int_{\mathbb{S}^{d-1}} D^{2 n_{o}} f(\vec{\pi})[w, \ldots, w] d \mathcal{H}^{d-1}
$$

Proof Since $4 d^{2}-\mathfrak{e}(\cdot)$ has a unique non-degenerate minimum at $\vec{\pi}$, the asymptotics of $\mathfrak{l}_{f}(z)$ as $z \searrow 4 d^{2}$ can be done along the lines of, for instance, [22,Lemma 4.1], hence, we skip the proof. 


\section{Remark A.3 When}

$$
|\widehat{v}(x)|=O\left(|x|^{2 n_{0}+d+1}\right) \quad \text { as }|x| \rightarrow \infty
$$

for some $n_{0} \geq 1$, one has $v \in C^{2 n_{0}}\left(\mathbb{T}^{d}\right)$. In this case the Taylor series of $f$ becomes only asymptotics of order $2 n_{0}-1$ and thus, instead of expansions (A.3)-(A.4) and (A.21)-(A.22) of $\mathfrak{l}_{f}$ one has only asymptotics up to order $2 n_{0}-1$.

\section{References}

1. Albeverio, S., Lakaev, S., Muminov, Z.: Schrödinger operators on lattices. The Efimov effect and discrete spectrum asymptotics. Ann. Inst. H. Poincaré Phys. Theor. 5, 743-772 (2004)

2. Albeverio, S., Lakaev, S., Makarov, K., Muminov, Z.: The threshold effects for the two-particle Hamiltonians on lattices. Commun. Math. Phys. 262, 91-115 (2006)

3. Andrew, A., Paine, J.: Correction of finite element estimates for Sturm-Liouville eigenvalues. Numer. Math. 50, 205-215 (1986)

4. Basti, G., Teta, A.: Efimov effect for a three-particle system with two identical fermions. Ann. Henri Poincaré 18, 3975-4003 (2017)

5. Ben-Artzi, M., Katriel, G.: Spline functions, the biharmonic operator and approximate eigenvalues. Numer. Math. 141, 839-879 (2019)

6. Boumenir, A.: Sampling for the fourth-order Sturm-Liouville differential operator. J. Math. Anal. Appl. 278, 542-550 (2003)

7. Damanik, D., Hundertmark, D., Killip, R., Simon, B.: Variational estimates for discrete Schrödinger operators with potentials of indefinite sign. Comm. Math. Phys. 238, 545-562 (2003)

8. Damanik, D., Teschl, G.: Bound states of discrete Schrödinger operators with super-critical inverse square potentials. Proc. Amer. Math. Soc. 135, 1123-1127 (2007)

9. Dipierro, S., Karakhanyan, A., Valdinoci, E.: A free boundary problem driven by the biharmonic operator. arXiv:1808.07696v2 [math.AP]

10. Egorova, I., Kopylova, E., Teschl, G.: Dispersion estimates for one-dimensional discrete Schrödinger and wave equations. J. Spectr. Theory 5, 663-696 (2015)

11. Graf, G., Schenker, D.: 2-magnon scattering in the Heisenberg model. Ann. Inst. Henri Poincaré Phys. Théor. 67, 91-107 (1997)

12. Graef, J., Heidarkhani, Sh., Kong, L., Wang, M.: Existence of solutions to a discrete fourth order boundary value problem. J. Difference Equ. Appl. 24, 849-858 (2018)

13. Gridnev, D.: Three resonating fermions in flatland: proof of the super Efimov effect and the exact discrete spectrum asymptotics. J. Phys. A: Math. Theor. 47 (2014)

14. Hiroshima, F., Lörinczi, J.: The spectrum of non-local discrete Schrödinger operators with a $\delta$-potential. Pacific J. Math. Industry 6, 1-6 (2014)

15. Hoffmann, S., Plonka, G., Weickert, J.: Discrete green's functions for harmonic and biharmonic inpainting with sparse atoms. In: X. Tai et al (eds) Energy Minimization Methods in Computer Vision and Pattern Recognition. EMMCVPR 2015. Lecture Notes in Computer Science, vol 8932 (2015). Springer, Cham

16. Jaksch, D., et al.: Cold bosonic atoms in optical lattices. Phys. Rev. Lett. 81, 3108-3111 (1998)

17. Kholmatov, Sh., Pardabaev, M.: On spectrum of the discrete bilaplacian with zero-range perturbation. Lobachevskii J. Math. 42, 1286-1293 (2021)

18. Klaus, M., Simon, B.: Coupling constant thresholds in nonrelativistic quantum mechanics. I. Shortrange two-body case. Ann. Phys. 130, 251-281 (1980)

19. Lakaev, S.: The Efimov effect of a system of three identical quantum lattice particles. Funkcional. Anal. Prilozhen. 27, 15-28 (1993)

20. Lakaev, S., Khalkhuzhaev, A., Lakaev, Sh.: Asymptotic behavior of an eigenvalue of the two-particle discrete Schrödinger operator. Theoret. Math. Phys. 171, 800-811 (2012)

21. Lakaev, S., Kholmatov, Sh.: Asymptotics of eigenvalues of two-particle Schrödinger operators on lattices with zero range interaction. J. Phys. A: Math. Theor. 44 (2011) 
22. Lakaev, S., Kholmatov, S.: Asymptotics of the eigenvalues of a discrete Schrödinger operator with zero-range potential. Izv. Math. 76, 946-966 (2012)

23. Luef, F., Teschl, G.: On the finiteness of the number of eigenvalues of Jacobi operators below the essential spectrum. J. Difference Equ. Appl. 10, 299-307 (2004)

24. Lewenstein, M., Sanpera, A., Ahufinger, A.: Ultracold Atoms in Optical Lattices. Simulating Quantum Many-Body Systems. Oxford University Press, Oxford (2012)

25. Mardanov, R., Zaripov, S.: Solution of Stokes flow problem using biharmonic equation formulation and multiquadrics method. Lobachevskii J. Math. 37, 268-273 (2016)

26. Mattis, D.: The few-body problem on a lattice. Rev. Mod. Phys. 58(2), 361-379 (1986)

27. McKenna, P., Walter, W.: Nonlinear oscillations in a suspension bridge. Arch. Rational Mech. Anal. 98, 167-177 (1987)

28. Mogilner, A.: Hamiltonians in solid-state physics as multiparticle discrete Schrödinger operators: problems and results. Adv. Sov. Math. 5, 139-194 (1991)

29. Naidon, P., Endo, S.: Efimov physics: a review. Rep. Prog. Phys. 80 (2017)

30. Rattana, A., Böckmann, C.: Matrix methods for computing eigenvalues of Sturm-Liouville problems of order four. J. Comput. Appl. Math. 249, 144-156 (2013)

31. Sobolev, A.: The Efimov effect. Discret. Spectr. Asymptotics. Commun. Math. Phys. 156, 127-168 (1993)

32. Tamura, H.: The Efimov effect of three-body Schrödinger operator. J. Funct. Anal. 95, 433-459 (1991)

33. Tee, G.: A novel finite-difference approximation to the biharmonic operator. Comput. J. 6, 177-192 (1963)

34. Yafaev, D.: On the theory of the discrete spectrum of the three-particle Schrödinger operator. Math. USSR-Sb. 23, 535-559 (1974)

35. Wall, M.: Quantum many-body physics of ultracold molecules in optical lattices. Models and simulation models. Springer Theses, Cham-Heidelberg-New York (2015)

36. Winkler, K., et al.: Repulsively bound atom pairs in an optical lattice. Nature 441, 853-856 (2006)

Publisher's Note Springer Nature remains neutral with regard to jurisdictional claims in published maps and institutional affiliations. 